\title{
Neuroprotective epi-drugs quench the inflammatory response and microglial/ macrophage activation in a mouse model of permanent brain ischemia
}

Mariana Mota ${ }^{1 \dagger}$, Vanessa Porrini $^{1^{\dagger \dagger}}$, Edoardo Parrella ${ }^{1 *} \mathbb{B}$, Marina Benarese ${ }^{1}$, Arianna Bellucci ${ }^{1}$, Sina Rhein ${ }^{2}$, Markus Schwaninger ${ }^{2}$ and Marina Pizzi ${ }^{1}$

\begin{abstract}
Background: Activation of NF-kappaB RelA deacetylated th the lysine residues, except the lysine 310, drives proapoptotic transcription in noxious brain ischemia. We showed that the sinergistic combination of the histone deacetilase inhibitor MS-275 with the sirtuin 1 activator resveratrol, at very low doses, restores normal RelA acetylation and elicit neuroprotection in mice subjected to transient middle cerebral artery occlusion (tMCAO) and primary cortical neurons exposed to oxygen-glucose-deprivation (OGD). The present study aims at corroborating the neuroprotective potential of the epigenetic treatment in a model of permanent brain ischemia and investigate its effect on post-ischemic inflammation and microglia activation.

Methods: Male mice subjected to permanent occlusion of the distal MCAO (pMCAO) were treated with vehicle or MS-275 $(20 \mu \mathrm{g} / \mathrm{kg})$ and resveratrol $(680 \mu \mathrm{gg} / \mathrm{kg})$ i.p. immediately after the ischemia. Microglia-containing mixed glial cultures were prepared from the brain of 1-3-day-old mice. Primary cortical neurons were prepared from 15-dayold embryonic mice.

Results: MS-275 and resveratrol in combination, but not individually, reduced infarct volume and neurological deficits evaluated $48 \mathrm{~h}$ after the pMCAO. At $24 \mathrm{~h}$, the treatment inhibited the RelA binding to Nos 2 promoter, reduced the elevated expression of Nos2, $116, \| 116, M r c 1$ and $Y m 1$ and the leukocytes infiltration in the ischemic area. The effect was nonpermanent. The treatment did not limit the sustained leukocyte infiltration or Nos 2 and $1 / 1 b$ transcription observed at 7 days. Though, it induced alternative activation markers of microglia/macrophages, Arg 1, Ym1 and Fcgr2b that could be added to Mrc1, Tgfb1 and Trem2 spontaneously increased at 7 days after ischemia. At 24 hours the drug treatment quenched the microglia/macrophages activation in the ischemic cortical sections, as shown by the recovered ramified morphology and lowered iNOS or CD68 immunoreactivity in Iba1-positive cells. Both microglia and astrocytes in mixed glial cultures, but not pure astrocytes, displayed signs of activation and iNOS-immunoreactivity when treated with a conditioned medium (NCM) from OGD-exposed cortical neurons. The (Continued on next page)
\end{abstract}

\footnotetext{
* Correspondence: e.parrella@unibs.it

${ }^{\dagger}$ Mariana Mota and Vanessa Porrini contributed equally to this work. 'Division of Pharmacology, Department of Molecular and Translational Medicine, University of Brescia, Viale Europa 11, 25123 Brescia, Italy Full list of author information is available at the end of the article
} changes were made. The images or other third party material in this article are included in the article's Creative Commons licence, unless indicated otherwise in a credit line to the material. If material is not included in the article's Creative Commons licence and your intended use is not permitted by statutory regulation or exceeds the permitted use, you will need to obtain permission directly from the copyright holder. To view a copy of this licence, visit http://creativecommons.org/licenses/by/4.0/. The Creative Commons Public Domain Dedication waiver (http://creativecommons.org/publicdomain/zero/1.0/) applies to the data made available in this article, unless otherwise stated in a credit line to the data. 
(Continued from previous page)
epigenetic drugs limited the OGD-NCM-mediated activation.

Conclusions: Our findings indicate that single treatment with MS-275 and resveratrol can reduce stroke-mediated brain injury and inflammation observed 2 days after the PMCAO and put the rational to test repeated administration of the drugs. The anti-inflammatory property of MS-275 and resveratrol combination can be ascribed to both primary direct inhibition of microglia/macrophage activation and secondary glial/macrophages inhibition mediated by neuroprotection.

Keywords: Stroke, Inflammation, Microglia, NF-kB RelA, Resveratrol, MS-275

\section{Background}

Acute ischemic stroke is a leading cause of death and disability worldwide $[1,2]$. This disease is characterized by damage to the brain tissue surrounding the occluded cerebral artery, most commonly the middle cerebral artery [3]. Treatment options are currently very limited, and the only available pharmacological treatment, recombinant tissue plasminogen activator (rtPA), has a very short 4.5 -hour time window, which benefits only about $5 \%$ of all stroke patients [4]. As such, there is a need for the development of new therapies that reduce post-ischemic neuronal injury.

The ischemic insult triggers a series of pathological processes, including excitotoxicity, apoptosis and inflammation, which contribute to cell death [5].

The nuclear factor-kappa B (NF-kB) family of transcription factors has diverse functions in the CNS. Under physiological conditions, NF- $\mathrm{kB}$ is a key regulator of cell survival, development, synaptic plasticity [6], and long-term memory [7]. By being a master regulator of the inflammatory and apoptotic processes, this transcription factor has also been associated with both acute and chronic neurodegenerative diseases, including, but not limited to, stroke, Alzheimer's, Parkinson's, and Huntington's diseases $[8,9]$. Five subunits compose the NF$\kappa B$ family of proteins: RelA (p65), RelB, c-Rel, p50, and $\mathrm{p} 52$, found in the cytoplasm as homo- or heterodimers. The activation of NF- $\mathrm{kB}$ plays both neuroprotective and neurotoxic roles depending on the subunits forming the transcription factor.

In particular, p50/RelA dimers are activated by neurotoxic stimuli, such as ischemia [10,11], glutamate [12], or $\beta$-amyloid [13]. This induces the transcription of proapoptotic factors, like Bim and Noxa [10], as well as the $1 \mathrm{~B}$ isoform of the divalent metal transporter-1 (1B/ DMT1), a membrane carrier responsible for iron accumulation and brain damage after injury [14].

We showed that p50/RelA exerts opposite effects depending on the acetylation state of RelA, and specifically on the acetylation on K310 $[15,16]$. In fact, either neuroprotective preconditioning or lethal ischemia activate p50/ RelA to the same extent. However, only in lethal oxygen and glucose deprivation (OGD) activated RelA shows a general deacetylation but a site-specific acetylation at the K310 residue, which favors pro-apoptotic transcription [15]. Acetylated RelA at K310 was found to interact with the CREB-binding protein (CBP) [16], detach from the anti-apoptotic $B c l-\chi_{L}$ promoter and bind to the proapoptotic Bim promoter [15].

In recent years, a significant body of data has shown that post-ischemic treatment with either histone deacetylase inhibitors (HDACi) [17-21] or the sirtuin activator resveratrol [22-25], is neuroprotective in experimental models of brain ischemia. We recently demonstrated that the combination of class I HDACi MS-275 (entinostat) with resveratrol, at very low doses, is able to correct the abnormal acetylation state of RelA, lowering the ratio K310/ total acetylation in models of transient brain ischemia and amyotrophic lateral sclerosis $[15,26]$. The combination of epigenetic drugs also restored the acetylation of $\mathrm{H} 3$ histones affected by lethal ischemia [15]. The synergistic action resulted from resveratrol-stimulated AMP-activated kinase (AMPK), which sustained the lysine acetilation by histone acetyltransferases (HATs) activity, upon HDAC inhibition by MS-275, and resveratrol-stimulated SIRT1 which targeted RelA K310 acetylation [15]. Concomitantly, the treatment induced a transcriptional switch and RelA binding from the pro- apoptotic Bim gene to antiapoptotic $B c l-\chi_{L}$ gene. In mice subjected to transient middle cerebral artery occlusion (tMCAO), MS-275 and resveratrol were neuroprotective when administered up to 7 hours after the insult. The beneficial effect was still evident after 72 hours [15].

With the aim to add translational power to the preclinical findings, we studied the effect of the epigenetic drugs in a model of permanent ischemic stroke that in comparison with the common models of transient ischemic stroke exhibits higher neuroinflammation and leukocyte infiltration [27].

Permanent occlusion of the middle cerebral artery (pMCAO) in mice produces an injury with good reproducibility of infarct size and neurological deficits [28]. Moreover, by mimicking stroke without reperfusion, it models the majority of clinical stroke cases [29]. MS-275 and resveratrol were tested in mice subjected to pMCAO to evaluate their anti-inflammatory potential 
and their capability to reduce infarct volume and promote neurologic recovery. The drug combination was also tested in primary mixed glial cultures incubated with a conditioned medium from OGD-exposed cortical neurons.

We here show that even in the pMCAO model the epigenetic drug combination of MS-275 and resveratrol limited the post-ischemic brain damage. Moreover, this treatment reduced the neuroinflammatory response associated with microglia activation, by directly quencing microglial reactivity.

\section{Methods}

\section{Drugs}

MS-275 and resveratrol were purchased from Vinci Biochem (Vinci, Italy) and Merck Millipore (Burlington, MA, USA), respectively. The compounds were dissolved in dimethylsulfoxide (DMSO, Sigma-Aldrich, Saint Louis, MO, USA).

\section{Animals}

Male C57BL/6NCrl mice (8- to 9-week old, 20-25 g, Charles River, Germany) were used. All experiments were performed according to the German animal protection law and were approved by the local animal welfare authorities (Ministerium für Energiewende, Landwirtschaft, Umwelt, Natur und Digitalisierung, Kiel, Germany). Before beginning any procedure, mice were housed for at least a week in ventilated cages in groups of four to five individuals in a $12 / 12$ hour light/dark cycle at $23^{\circ} \mathrm{C}$, with ad libitum access to food and water.

\section{pMCAO stroke model}

To induce ischemic stroke, mice were subjected to permanent occlusion of the distal MCA [30]. Briefly, mice were anaesthetized with tribromoethanol $(15 \mu \mathrm{L}$ of $2.5 \%$ tribromoethanol/g body weight, i.p.) and an incision was made between the ear and the orbit on the left side. The temporal muscle was removed and a burr hole was drilled to expose the MCA, which was then occluded by bipolar electrocoagulation (Model ICC 50, Erbe, Marietta, GA, USA). The surgery was done under a microscope (Hund) and rectal temperature was maintained at $37^{\circ} \mathrm{C}$ during surgery by a heating pad. After the incision was closed by a suture, mice were placed under a heating lamp until fully recovered. Animals undergoing sham surgery went through the same surgical procedures, but without occlusion of the MCA. Mice were randomized to the treatment groups. MS-275 and resveratrol were dissolved in saline with $1 \%(\mathrm{v} / \mathrm{v})$ DMSO at the doses of $20 \mu \mathrm{g} / \mathrm{kg}$ and $680 \mu \mathrm{g} / \mathrm{kg}$, respectively, and intraperitoneally administered immediately after the pMCAO procedure. Mortality was $<15 \%$ and did not differ between treatment groups.

\section{Behaviour analysis}

To evaluate sensorimotor function, two established tests were used: the corner and latency-to-move tests [30]. Briefly, in the corner test, mice were allowed to enter a $30 \times 20 \mathrm{~cm}$ corner with an angle of $30^{\circ} 24 \mathrm{~h}$ before (considered as the baseline value) and $48 \mathrm{~h}$ after pMCAO, and the number of right and left turns on rearing out of 12 trials were counted, or for a maximum of 30 minutes. For the latency-to-move test, mice were placed at the center of a plain board. The time to cross one body length $(7 \mathrm{~cm})$ was measured three times for each mouse, $24 \mathrm{~h}$ before and $48 \mathrm{~h}$ after pMCAO.

\section{Infarct volume}

Forty-eight hours after pMCAO, mice were deeply reanaesthetized with tribromoethanol and intracardially perfused with Ringer's solution. Brains were removed and coronally cryosectioned (20- $\mu \mathrm{m}$ thick) every 400 $\mu \mathrm{m}$. Coronal sections were stained with a silver technique as described previously [31]. Stained sections were scanned at $600 \mathrm{dpi}$ and the infarct area was measured. The total infarct volume was obtained from integrating infarcted areas after subtracting the difference between the area of the ischemic and the non-ischemic hemispheres to correct for brain edema [30].

\section{Real-time quantitative reverse transcription-polymerase chain reaction (qRT-PCR)}

Analysis of mRNA expression of inflammatory markers was performed 1 and 7 days day after pMCAO. Total RNA was purified from ipsilateral hemispheres using the RNeasy Mini Kit for total RNA extractions (Qiagen, Hilden, Germany), according to the manufacturer's instructions. Then, $1 \mu \mathrm{g}$ of total RNA was transcribed to cDNA using the Quantitect ${ }^{\circ}$ Reverse Transcription Kit (Qiagen, Hilden, Germany) with optimized mix of oligo-dT and random primers as primer. The following primers were used for quantitative RT-PCR: Arg1 forward GTGTACATTGGCTTGCGAGA, Arg1 reverse AATCGGCCTTTTCTTCCTTC, Fcgr3 forward TATCGGTGTCAAATGGAGCA, Fcgr3 reverse GCACCT TAGCGTGATGGTTT, $F c g r 2 b$ forward AGGGCCTCCA TCTGGACTG, Fcgr2b reverse GTGGTTCTGGTAATCA TGCTCTG, Gapdh forward TCAACAGCAACTCCCACT CTT, Gapdh reverse CCAGGGTTTCTTACTCCTTGG, Il6 forward CCTACCCCAATTTCCAATGCT, Il6 reverse TATTTTCTGACCACAGTGAGGAAT, $\quad l l l b$ forward GGCTTCAGGCAGGCAGTATC, $I l 1 b$ reverse TAATGG GAACGTCACACACC, Nos2 forward AGCCAAGCCC TCACCTACTT, Nos2 reverse GTGGGGTTGTTGCTGA ACTT, Mrc1 forward AAGGTTCGGGATTGTGGAG, $M r c 1$ reverse TCGTAGTCAGTGGTGGTTCC, Tgfb1 forward CAATTCCTGGCGTTACCTTG, Tgfb1 reverse GGTTCATGTCATGGATGGTG, Trem2 forward CACT CTGAAGAACCTCCAAGC, Trem2 reverse ATTCCTGG 
AGGTGCTGTGTT, $Y m 1$ forward GCCCACCAGGAAAG TACACA, Ym1 reverse CACGGCACCTCCTAAATTGT.

cDNA $(1-5 \mu \mathrm{L})$ was amplified with $\mathrm{iQ}^{\mathrm{m}}$ SYBR Green Supermix (Bio-Rad, Hercules, CA, USA) and forward and reverse primers $(10 \mu \mathrm{M})$. Each reaction was performed in triplicate, using the fast program in ViiA 7 Real-Time PCR System (Applied Biosystems, Foster City, CA, USA). For standardization of quantification, Gapdh was used as housekeeping gene. Data were analyzed following the comparative Ct method, where dCt: Housekeeping Ct Target Ct; and ddCt: Calibrator dCt - Sample dCt.

\section{Chromatin immunoprecipitation and real-time PCR analysis}

Chromatin immunoprecipitation (ChIP) assays were performed to study RelA interactions and H3 histone acetylation at the Nos 2 and Il6 promoters in ipsilateral hemispheres of mice one day after pMCAO, using a SimpleChIP ${ }^{\oplus}$ Enzymatic Chromatin IP Kit (Magnetic Beads) (\#9003S, Cell Signaling Technology, Danvers, MA, USA) as previously described [15], with some modifications. Briefly, frozen brain tissue from the ipsilateral hemispheres of 2 animals per group were pooled and chopped into small pieces. Proteins were crosslinked to DNA using $1.5 \%$ formaldehyde (Sigma-Aldrich, St. Louis, MO, USA). The crosslinked chromatin was extracted using buffers A and B provided in the kit, digested by micrococcal nuclease, and sonicated on ice with an ultrasonic homogenizer (5 cicles, $20 \mathrm{sec}$, 50\% power output, Bandelin SONOPLUS, Berlin, Germany). Chromatin was immunoprecipitated with antibodies anti-acetyl H3(K9/18) (Upstate-Millipore \#07-593, Burlington, MA, USA), anti-RelA (Santa Cruz Biotechnology \#sc-372X, Dallas, TX, USA) and anti-IgG (negative control), overnight at $4^{\circ} \mathrm{C}$ with gentle rotation. The complexes were captured by magnetic-coupled protein $\mathrm{G}$ beads for $2 \mathrm{~h}$ at $4{ }^{\circ} \mathrm{C}$ with gentle rotation. Following washing, bound DNA fragments were eluted and analyzed by subsequent real-time qRT-PCR using the following primers: Nos2 forward CCACAGAGTGATGTAATCAAGCA, Nos2 reverse GCAGCAGCCATCAG GTATTT, Il6 forward CCCACCCTCCAACAAAGATT, Il6 reverse TGAGCTACAGACATCCCCAGT. Immunoprecipitated DNA $(3 \mu \mathrm{l})$ was amplified with $\mathrm{iQ}^{\mathrm{sm}}$ SYBR Green Supermix (Bio-Rad, Hercules, CA, USA) and forward and reverse primers $(10 \mu \mathrm{M})$. Each reaction was performed in triplicate, using the fast program in ViiA 7 Real-Time PCR System (Applied Biosystems, Foster City, CA, USA). Ct values obtained by qRT-PCR analysis of samples immunoprecipitated with anti-RelA or antiacetyl-H3 antibodies were normalized over corresponding $\mathrm{Ct}$ values obtained by IgG immunoprecipitation, and further normalized over relative $\mathrm{Ct}$ values obtained in INPUT (no immunoprecipitated) chromatin. Final data obtained in mice subjected to pMCAO or pMCAO plus treatment were normalized to the data obtained in sham operated mice.

\section{Immunohistochemistry}

One or 7 days after pMCAO, mice were anaesthetized with tribromoethanol and perfused transcardially with Ringer's solution and 4\% paraformaldehyde (PFA) in phosphate buffered saline (PBS). Brains were removed and postfixed in $4 \%$ PFA at $4{ }^{\circ} \mathrm{C}$ for $2 \mathrm{~h}$, followed by cryoprotection in $30 \%$ sucrose in $\mathrm{PBS}$ overnight $(\mathrm{O} / \mathrm{N})$ at $4^{\circ} \mathrm{C}$. After snapfreezing in isopentane (Sigma-Aldrich, St. Louis, MO, USA) cooled in liquid nitrogen, $15-\mu \mathrm{m}$-thick coronal cryosections were mounted on Superfrost Plus slides and preserved at $-80^{\circ} \mathrm{C}$ until use.

For double immunofluorescence staining, tissues were permeabilized with $0.3 \%$ Triton X-100 (Sigma-Aldrich, St. Louis, MO, USA) in $20 \%$ methanol in PBS for $30 \mathrm{~min}$ and then blocked with PBS containing 3\% bovine serum albumine (BSA) (Sigma-Aldrich, St. Louis, MO, USA), $2 \%$ normal goat serum (Sigma-Aldrich, St. Louis, MO, USA) for $2 \mathrm{~h}$ at RT. Sections were incubated overnight at $4{ }^{\circ} \mathrm{C}$ with the first primary antibody in blocking solution: mouse anti-iNOS (1:50, BD Biosciences \#610329, Franklin Lakes, NJ, USA), rat anti-CD68 (1:100, Bio-Rad \#MCA1957, Hercules, CA, USA), or rat anti-MRC1 (1: 100, Bio-Rad \#MCA2235, Hercules, CA, USA). The following day, slices were incubated with the secondary antibody for $1 \mathrm{~h}$ at RT in PBS containing 1\% BSA and 0.3\% Triton X-100: for iNOS, biotinylated goat antimouse (1:600, Vector laboratories \#BA-9200, Burlingame, CA, USA), or for CD68 and MRC1, biotinylated goat anti-rat (1:600, Vector laboratories \#BA-9401, Burlingame, CA, USA) followed by streptavidin-Alexa Fluor 594 (1:1500, ThermoFisher Scientific \#S11227, Waltham, MA, USA). Then, sections were incubated $2 \mathrm{~h}$ at $37^{\circ} \mathrm{C}$ with the second primary antibody, rabbit anti-Iba1 (1: 1000, Wako \#019-19741, Osaka, Japan), in blocking solution. Slices were incubated in the second secondary antibody, goat anti-rabbit Alexa Fluor 488 (1:1500, Jackson ImmunoResearch \#111-545- 144, West Grove, PA, USA), for $1 \mathrm{~h}$ at RT in PBS containing 1\% BSA and $0.3 \%$ Triton X-100. Finally, sections were incubated with Hoechst 33342 (1 mg/mL, Sigma-Aldrich, St. Louis, MO, USA) for 3 minutes to stain cellular nuclei and mounted with Vectashield (Vector Laboratories, Burlingame, CA, USA).

3,3'-Diaminobenzidine (DAB) immunostaining tissues were permeabilized with $0.3 \%$ Triton X-100 (Sigma-Aldrich, St. Louis, MO, USA) in 20\% methanol in PBS for $20 \mathrm{~min}$ and then blocked with PBS containing 5\% BSA (Sigma-Aldrich, St. Louis, MO, USA) for $2 \mathrm{~h}$ at RT. Slices were incubated overnight at $4^{\circ} \mathrm{C}$ with the primary antibody anti- CD45 (1:70 Bio-Rad \#MCA1031, 
Hercules, CA, USA) in blocking solution. The following day, brain sections were incubated with secondary antibody, biotinylated goat anti-rat (1:500, Vector laboratories \#BA-9401, Burlingame, CA, USA), for $1 \mathrm{~h}$ at RT in PBS containing $1 \%$ BSA and $0.3 \%$ Triton X-100. Finally, the signal was visualized by avidin-biotin-horseradish peroxidase technique (ABC Elite; Vector Laboratories, Burlingame, CA, USA) using ImmPACT ${ }^{\mathrm{Tm}}$ DAB kit (Vector Laboratories, Burlingame, CA, USA) as chromogen.

Quantification of CD45 immunoreactivity was performed on digitized images using ImageJ software [32]. Briefly, brains from 3 mice (3-4 sections from each mouse) were analyzed by examining an average of 4 fields per section. Data were assessed as CD45 positive pixels in the ipsilateral hemisphere/CD45 positive pixels in the contralateral hemisphere and indicated as CD45 staining percentage area (ipsilateral/contralateral).

\section{Primary cell cultures}

Primary cell cultures were obtained from $\mathrm{C} 57 \mathrm{Bl} / 6$ mice (Charles River, Calco, Italy). The animal studies were approved by the Animal-welfare body of the University of Brescia and were in accordance with the Directive 2010/ 63/EU on the protection of animals used for scientific purposes. In details, primary cultures of cortical neurons were prepared from 15-day-old embryonic mice and cultured as previously described [33]. The cells were seeded at the density of $2 \times 10^{6}$ cells in $21 \mathrm{~cm}^{2}$ culture dishes (Nunc, Germany) using Neurobasal medium (Invitrogen Corporation, Carlsbad, CA, USA) supplemented with $2 \%$ B27 (Invitrogen Corporation, Carlsbad, CA, USA), $0.5 \mathrm{mM} \mathrm{L-}$ glutamine (Euroclone, Pero, Italy) and $50 \mathrm{U} / \mathrm{mL}$ penicillin/streptomycin (Euroclone, Pero, Italy). The neurons were used after 11 days in vitro (DIV). Mixed glial cell cultures were prepared from the brains of 1-3-day-old mice [34]. The cells were grown in 24-well-plates at the density of $1.2 \times 10^{5}$ cells per well using DMEM medium supplemented with $10 \%$ fetal bovine serum (FBS) (Euroclone, Pero, Italy), $4 \mathrm{mM}$ L-glutamine (Euroclone, Pero, Italy) and $200 \mathrm{U} / \mathrm{ml}$ penicillin/streptomycin (Euroclone, Pero, Italy). Mixed glial cells were used after 14 DIV. For immunocytochemistry (ICC) studies, a coverslip was included in the well. Approximately 10\% of microglia was present in the mixed microglia-astrocyte cultures. Pure astrocytes were isolated by shaking the flasks containing the mixed glia cells for 6 hours [35]. They were seeded in 24-well-plates at the density $1.2 \times 10^{5}$ cells per well and used after 14 DIV. For ICC experiments the cells were grown on coverslips.

\section{Oxygen and glucose deprivation}

Primary cultures of mouse cortical neurons were exposed to OGD for $3 \mathrm{~h}$ according to previous experiments [33]. Briefly, cells were incubated with deoxygenated glucose-free balanced salt solution and transferred to an air-tight chamber fluxed with $95 \% \mathrm{~N}_{2}$ and $5 \% \mathrm{CO}_{2}$ for 10 min to reach an $\mathrm{O}_{2}$ concentration lower than $0.4 \%$. A parallel set of cultured neurons were incubated for $3 \mathrm{~h}$ with normally oxygenated, balanced salt-solution containing glucose and used as a control. After the anoxic insult, cortical neurons were transferred to recover in neurobasal medium containing $0.4 \%$ B27 supplement.

\section{Preparation of neuron-derived conditioned medium and measurement of lactate dehydrogenase release}

Neuron-derived Conditioned Medium (NCM) was prepared by diluting 1:5 in DMEM medium the medium collected from primary neuronal cultures subjected to 3 h OGD and $24 \mathrm{~h}$ recovery (NCM-OGD) or from neurons that underwent the same protocol except OGD (NCM-control). Primary mixed glia and pure astrocytes cultures were then incubated with NCM overnight. The duration of NCM incubation was chosen in order to detect cellular damage but no major cell death. Glial cell cultures were treated with MS-275 $(0.1 \mu \mathrm{M})$ and resveratrol $(3 \mu \mathrm{M})$, or vehicle, concomitantly with NCM incubation. At the end of NCM exposition period, we estimated the cellular injury by assessing the ratio between the amount of lactate dehydrogenase (LDH) released in culture medium and the total releasable $\mathrm{LDH}$ using the CytoTox $96^{\circ}$ Non-Radioactive Cytotoxicity Assay (Promega, Fitchburg, WI, USA).

\section{Immunocytochemistry}

Double immunofluorescence staining for Iba1 and GFAP was initiated by performing permeabilization and blocking, followed by an incubation at room temperature for $2 \mathrm{~h}$ with the primary antibody rabbit anti-Iba1 (1:900, Wako \#019-19741, Osaka, Japan), and then by a 1-h incubation at room temperature with the secondary antibody goat anti-rabbit Alexa Fluor 488 (1:800, Jackson ImmunoResearch \#111-545- 144, West Grove, PA, USA). Then, cells were incubated overnight at $4^{\circ} \mathrm{C}$ with the second primary antibody mouse anti-GFAP (1:200, Sigma-Aldrich \#G3893 St. Louis, MO, USA) and then the second secondary antibody goat anti-mouse Cy3 (1: 500, Jackson ImmunoResearch \#115-165-003, West Grove, PA, USA) for $1 \mathrm{~h}$ at room temperature. Double immunofluorescence staining for Iba1 and iNOS was initiated by performing permeabilization and blocking, followed by an incubation at room temperature for $2 \mathrm{~h}$ with the primary antibody rabbit anti-Iba1 (1:900, Wako \#019-19741, Osaka, Japan), and then by a 1-h incubation at room temperature with the secondary antibody goat anti-rabbit Alexa Fluor 488 (1:800, Jackson ImmunoResearch \#111-545- 144, West Grove, PA, USA). Then, cells were incubated overnight at $4^{\circ} \mathrm{C}$ with the second primary antibody mouse anti-iNOS (1:25, BD 
Biosciences \#610329, Franklin Lakes, NJ, USA) followed by a 1-h incubation at room temperature in the second secondary biotinylated goat anti-mouse (1:600, Vector laboratories \#BA-9200, Burlingame, CA, USA), followed by streptavidin-594 (1:2000, ThermoFisher Scientific \#S11227, Waltham, MA, USA).

\section{Microscopy}

Immunostained brain sections and cells were observed with an inverted light/epifluorescence microscope (Olympus IX50; Olympus Corporation, Tokyo, Japan), equipped with a digital camera (Olympus XC 30, Olympus Corporation, Tokyo, Japan) and cellSens Software (Olympus Corporation, Tokyo, Japan). Captured images were adjusted for brightness and contrast with Adobe Photoshop (Adobe system, San Jose, CA, USA) software.

\section{Statistical analysis}

Comparisons between two goups were performed using the parametric Student's t-test or the non parametric Mann Whitney test. The comparison between more than two groups, where $n$ was too small for D'Agostino-Pearson test of normality, was made using Kruskal-Wallis one-way analysis of variance with Dunn's multiple comparison test. For comparison of normally distributed data from more than two groups, 1-way or 2-way ANOVA followed by Bonferroni post hoc test were employed. Data are presented as mean \pm s.e.m. $\mathrm{P}<0.1$ was considered as a trend, and $\mathrm{P}<0.05$ as significant. All data were analyzed using GraphPad Prism (GraphPad Software, San Diego, CA, USA).

\section{Results}

Treatment with MS-275 and resveratrol reduced infarct size and neurological deficits two days after PMCAO

The mouse model of permanent ischemia was accomplished by electrocoagulating the MCA. Treatments were administered i.p. immediately after the end of the procedure. To determine the synergistic protective effect of MS-275 plus resveratrol, the two drugs were injected individually (MS-275, $20 \mu \mathrm{g} / \mathrm{kg}$; resveratrol, $680 \mu \mathrm{g} / \mathrm{kg}$ ), or in combination (MS-275 $20 \mu \mathrm{g} / \mathrm{kg}+$ resveratrol 680 $\mu \mathrm{g} / \mathrm{kg})$. We previoulsy showed that these doses were able to sinergistically reduce infarct volume and neurological deficits in mice subjected to tMCAO [15]. Experiments to assess the outcome of experimental stroke were conducted 2 days after pMCAO. After the permanent ischemia, infarcts were significantly smaller in mice treated with the combination of MS-275 $(20 \mu \mathrm{g} / \mathrm{kg})$ and resveratrol $(680 \mu \mathrm{g} / \mathrm{kg})$ than in vehicle- and single drug-treated animals (Fig. 1a). In parallel, only the treatment with the drug combination improved the pMCAO-induced neurological deficits as shown by the corner test. In 12 trials, mice tended to turn more often to the contralateral, that is right, than to the ipsilateral side after pMCAO. The combination of MS-275 and resveratrol normalized this preference for the right side observed in vehicle-, MS-275- and resveratrol-treated groups (Fig. 1b). Additionally, after pMCAO, the latency-to move one body length was prolonged. Only the treatment with the combination of MS-275 and resveratrol significantly improved this parameter (Fig. 1c).

\section{Treatment with MS-275 and resveratrol reduced the inflammatory response after pMCAO}

In order to study the effect of MS-275 and resveratrol on the early inflammatory status following pMCAO, ipsilateral hemispheres were analyzed for mRNA expression of inflammatory markers 1 day after a single postinsult administration of the drugs. The transcripts of several important players of the inflammatory and microglial/macrophage response in cerebral ischemia were analysed (Fig. 2). IL-6 (Il6) and IL-1ß (Il1b) are two crucial pro-inflammatory cytokines in experimental and human stroke [36]. CD16 (Fcgr3) and CD32 (Fcgr2b) are surface receptors for immunocomplexes associated with activated microglia/macrophages where $F c g r 2 b$ acts by driving the polarization of microglia/macrophages toward an anti-inflammatory phenotype [37]. iNOS (Nos2), via the production of NO, is an important cytotoxic enzyme in cerebral ischemia [38]. MRC1 (Mrc1), Ym1 (Ym1) and Arg1 (Arg1) are markers of alternatively activated microglia and macrophages [39]. TGF- $\beta 1$ $(T g f b 1)$ is one of the main contributors to the antiinflammatory environment after ischemic brain damage [38]. TREM2 (Trem2) is a receptor associated with the phagocytic activity of microglia [40]. qRT-PCR analysis of ipsilateral hemispheres showed a general increase of inflammation and the activation of microglia/macrophages 1 day after pMCAO. Permanent MCAO significantly induced the transcription of Il6, Nos2, Illb, Mrc1 and $Y m 1$ (Fig. 2a-c, f, h), and caused an upward trend in the Arg1 expression (Fig. 2g). The expression of Fcgr3, Fcgr2b, Tgfb1 and Trem 2 remained unchanged (Fig. 2d, e, i, l). When compared to vehicle, the treatment with MS-275 $(20 \mu \mathrm{g} / \mathrm{kg})$ and resveratrol $(680 \mu \mathrm{g} / \mathrm{kg})$ globally prevented the inflammatory response by reducing the expression of Nos2, Il1b, Il6 (Fig. 2a-c) as well as the microglia/macrophage alternative activation markers Mrc1 and Ym1 (Fig. 2f, g).

When analized in mice 7 days after the MCAO, either pro- or anti-inflammatory transcripts appeared elevated in comparison with the sham condition (additional file 1 ). With the exception of reduced Il6 transcription, the expression of pro-inflammatory Nos2 and Fcgr3 increased, while $I l 1 b$ and Fcgr $2 b$ maintained a trend of increase. In line with a progressive elevation of the antiinflammatory/neuroprotective response of microglia and 


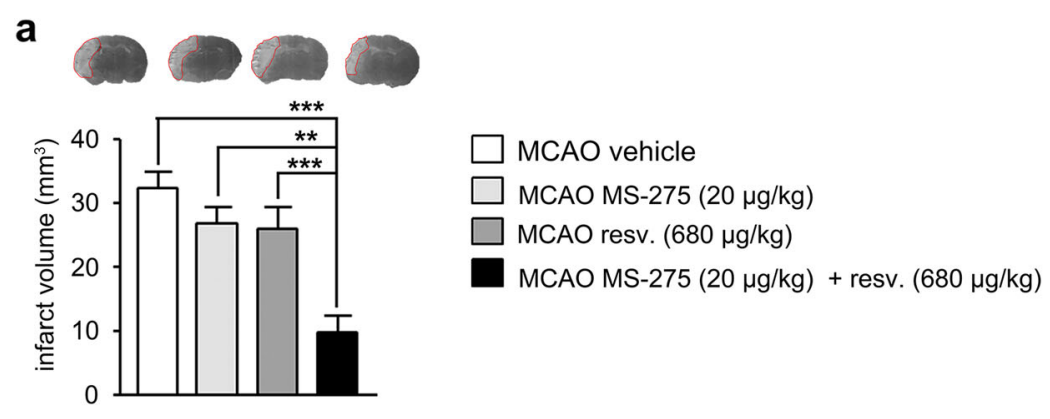

b

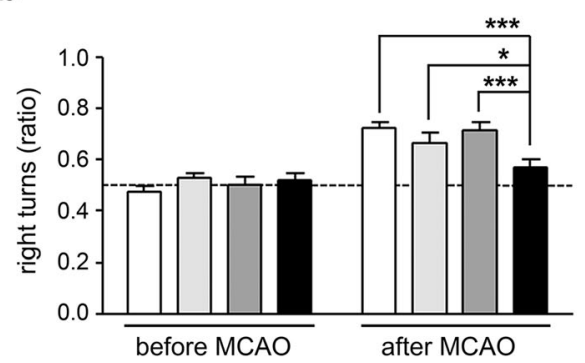

C

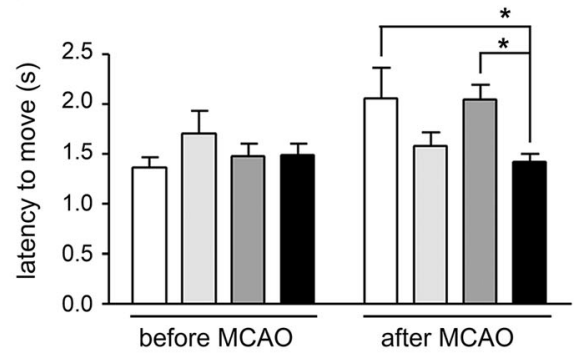

Fig. 1 The combination of MS-275 and resveratrol is neuroprotective two days after pMCAO. (a) Treatment with MS-275 (20 $\mu \mathrm{g} / \mathrm{kg})$ and resveratrol $(680 \mu \mathrm{g} / \mathrm{kg})$ reduced the infarct volume, determined 2 days after pMCAO. Silver-stained coronal brain sections showing infarcts in light grey are depicted on top of the panel. ANOVA, ${ }^{* *} P<0.01{ }^{* * *} P<0.001$ (Bonferroni post hoc test). Values are means \pm s.e.m. ( $n=9-11$ from five independent experiments). (b) In the corner test, vehicle-, MS-275- and resveratrol-treated mice showed a preference to turn to the right side 2 days after pMCAO. Treatment with MS-275 and resveratrol reduced this preference for right turns. The dashed line indicates the expected behaviour without a side preference. Two-way repeated-measures ANOVA, ${ }^{*} P<0.05$, ${ }^{* * *} P<0.001$ (Bonferroni post hoc test). Values are means \pm s.e.m. ( $n=11-13$ from five independent experiments). (c) Treatment with MS-275 and resveratrol improved the latency to move that was increased 2 days after pMCAO. Twoway repeated-measures ANOVA, ${ }^{*} P<0.05$ (Bonferroni post hoc test). Values are means \pm s.e.m. $(n=11-13$ from five independent experiments).

infiltrating macrophages in the post stroke period [27, 32, 41, 42], at 7 days Mrc1, Tgfb1 and Trem 2 transcripts also significantly increased. At this time, the inhibitory effect of MS-275 and resveratrol was no longer evident on pro-inflammatory signals. Though the drug combination further boosted the expression of markers for microglia/macrophage alternative activation state by elevating transcription of $F g c r 2 b$ together with $Y m 1$ and Arg1 (additional file 1).

\section{Treatment with MS-275 and resveratrol decreased the} binding of RelA to Nos2 promoter one day after pMCAO

Nos 2 and Il6, two pro-inflammatory genes modified by MS-275 and resveratrol treatment, are target of NF-kBmediated transcriptional regulation [43]. We analysed the interactions between RelA and Nos 2 or Il6 promoters in the cortex of mice 1 day after pMCAO, as well as the capability of the treatment with MS-275 $(20 \mu \mathrm{g} / \mathrm{kg})$ and resveratrol $(680 \mu \mathrm{g} / \mathrm{kg})$ to modulate that interaction. After chromatin immunoprecipitation with antibodies for Ac$\mathrm{H} 3(\mathrm{~K} 9 / 18)$ and RelA, the relative level of Nos 2 and Il6 promoters associated with acetylated H3 histone and bound RelA was determined by real-time qRT-PCR analysis, using primers that amplified the sequence of the Il6 and Nos 2 promoters that include the $\mathrm{\kappa B}$ binding sites.
One day after pMCAO, both RelA binding and $\mathrm{H} 3$ acetylation at the Nos 2 promoter increased (Fig. 3a, b), whilst both remained unchanged at the $I l 6$ promoter (Fig. 3c, d). It suggests that the acetylation of $\mathrm{H} 3$ histone close to $\mathrm{kB}$ binding site was driven by the RelA binding to the promoter. When compared to vehicle, the treatment with MS-275 and resveratrol decreased the binding of RelA and the acetylation of $\mathrm{H} 3$ histone at the Nos 2 promoter, but only slightly decreased those at the Il6 promoter (Fig. 3a-d).

\section{Treatment with MS-275 and resveratrol reduced leukocytes infiltration and microglia/macrophage inflammatory profile}

Analysis of infiltrating leukocytes was carried out by evaluating CD45 immunoreactivity (Fig. 4). One day after MCAO, the CD45-positive cells significantly increased in the ipsilateral penumbra area when compared to the contralateral hemisphere (Fig. 4a). MS-275 and resveratrol reduced the leukocyte infiltration, in line with the anti-inflammatory effect induced by the treatment (Fig. 4a, b). This positive effect was lost at 7 days where the higher leukocyte infiltration in ipsilateral ischemic emisphere appeared elevated in mice treated with MS- 

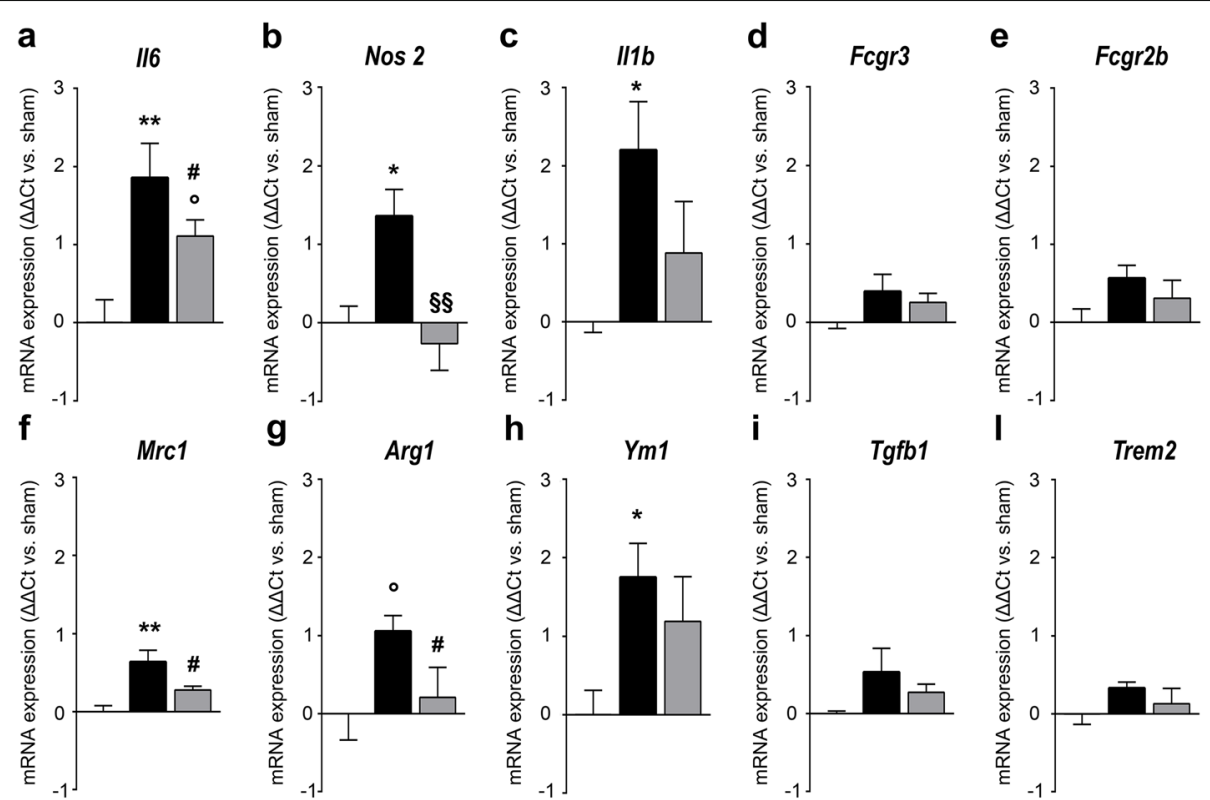

sham vehicle

MCAO vehicle

$\square$ MCAO MS-275 $(20 \mu \mathrm{g} / \mathrm{kg})+$ resv. $(680 \mu \mathrm{g} / \mathrm{kg})$

Fig. 2 Inflammatory and microglia/macrophage mRNA expression profile in the ipsilateral hemisphere 1 day after pMCAO. (a-I) Quantification of II6, Nos2, II1b, Fcgr3, Fcgr2b, Mrc1, Arg1, Ym1, Tgfb1 and Trem2 by qRT-PCR in the ipsilateral hemisphere 1 day after pMCAO. Following pMCAO, the expression of (a) 116 , (b) Nos2, (c) II16, (f) Mrcl and (h) Ym1 was higher, and (g) Argl showed an upward trend, if compared to the sham vehicle group. Treatment with MS-275 and resveratrol promoted a mild reduction of the expression of (a) 116 , (f) Mrcl and (g) Arg1, and prevented the increase of (b) Nos2, (c) I/1b and (h) Ym1 transcript levels. One-way ANOVA followed by Holm-Šídák test, ${ }^{\circ}$ trend, ${ }^{*} p<0.05$, ${ }^{*} p<0.01$ vs. sham vehicle; ${ }^{\# t r e n d}{ }^{\S \S} p<0.001$ vs. MCAO vehicle. Data (means \pm s.e.m., $n=6$ ) are expressed as fold changes over values obtained in ipsilateral hemisphere from sham operated mice.

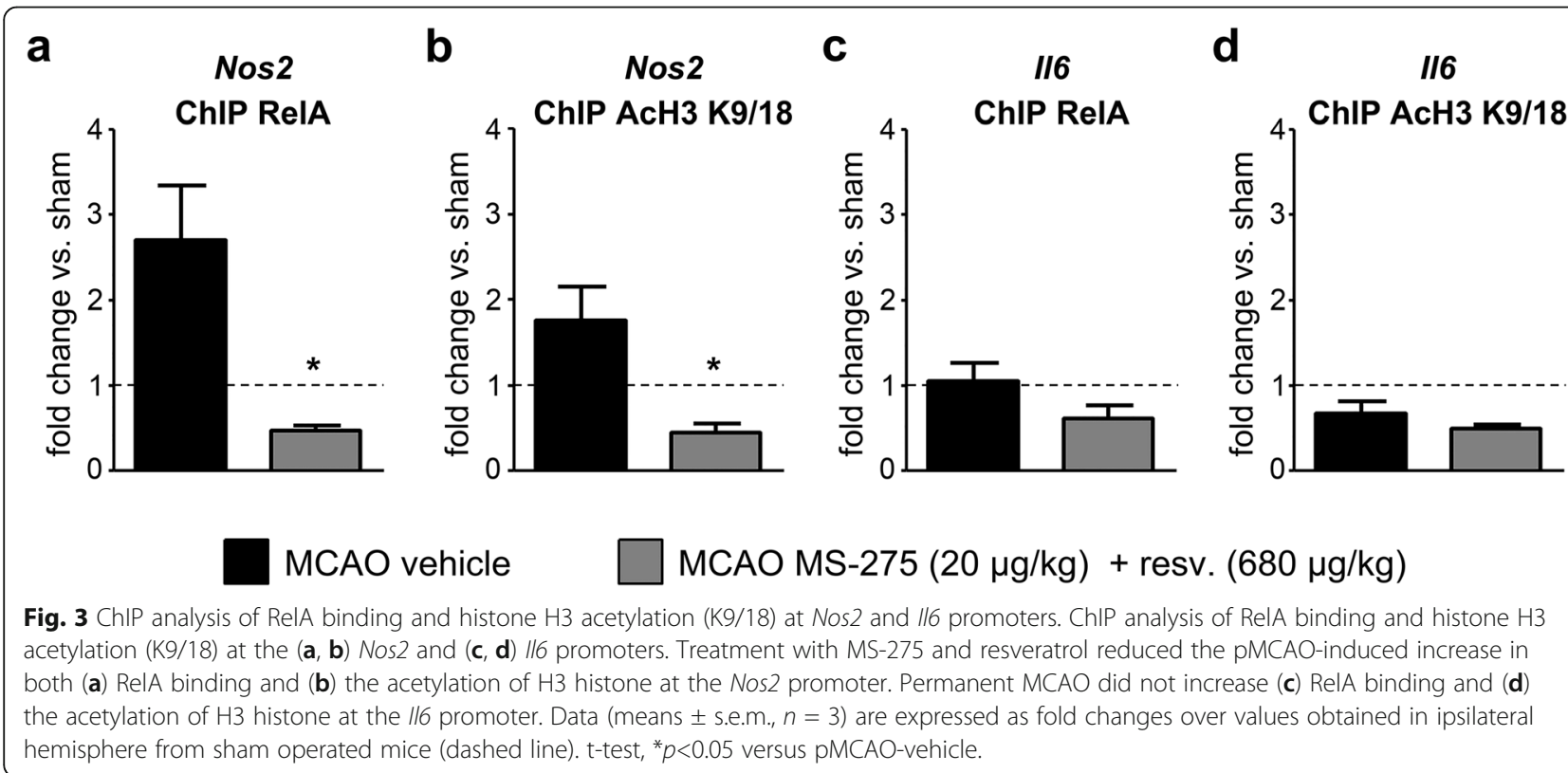


275 and resveratrol as in vehicle-treated MCAO mice (additional file 2).

When analysing tissue from ipsilateral hemispheres, the results of real-time qRT-PCR for inflammatory genes cannot distinguish changes in single cell types. After the surprising finding that MS-275 and resveratrol treatment decreased pro- and anti-inflammatory transcripts 1 day after pMCAO, we evaluated the pro-inflammatory activation state of microglia/macrophages in the ischemic brains using their common markers Ibal and iNOS. One day after pMCAO, the morphology of Iba1+ cells was analysed in the peri-infarct area of the ipsilateral hemisphere. Permanent ischemia induced the activation of microglia/macrophages that acquired a bushier, less ramified morphology (Fig. 5e, Fig. 6e, additional file 3e, cells marked with \#). Conversely, Iba1+ cells from MS275 and resveratrol-treated mice recovered a more ramified shape, similar to that displayed by the sham group of mice (Fig. 5f, Fig. 6f, additional file 3f, cells marked with /). Immunofluorescence for the pro-inflammatory marker iNOS was increased 1 day after pMCAO, compared with sham animals (Fig. 5b).

The treatment with MS-275 and resveratrol decreased the reactivity to iNOS (Fig. 5c). Not all iNOS+ cells were positive for Iba1, which is consistent with the fact that other cells, such as astrocytes and neurons, express iNOS and can contribute to the observed increased transcription [44] (Fig. 5g-i).

CD68 is a lysosomal glycoprotein associated with an increased phagocytic activity [45]. In control conditions, microglia do not express CD68, while after brain ischemia, activated microglia have been found positive for CD68 [46, 47]. One day after pMCAO, the CD68 immunoreactivity increased in cortical section, and the treatment with MS-275 and resveratrol lowered the CD68 signal (Fig. 6a-c). Some of the CD68+ cells were also Iba1+, but others negative (Fig. 6g-i). The latter population, CD68+/Iba1- cells, could very well be neutrophils known to infiltrate the brain $24 \mathrm{~h}$ after pMCAO [27, 47, 48], as they are Iba1- cells endowed with phagocytic capacity and positivity to CD68 [47]. The immunoreactivity to MRC1 remained unchanged in all three experimental groups: sham, vehicle, and treated (additional file 3a-i). No detectable immunoreactivity for iNOS, CD68, or MRC1 was evident in the contralateral hemisphere of all animal groups.

Taken together, these data showed that the treatment with the combination of MS-275 and resveratrol diminished the pro-inflammatory activation state of microglia/ macrophages. It was associated with a reduced reactivity a

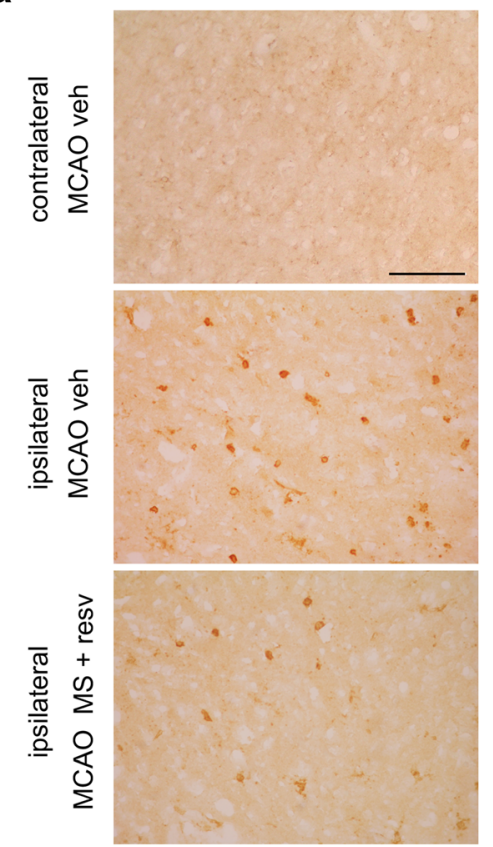

b

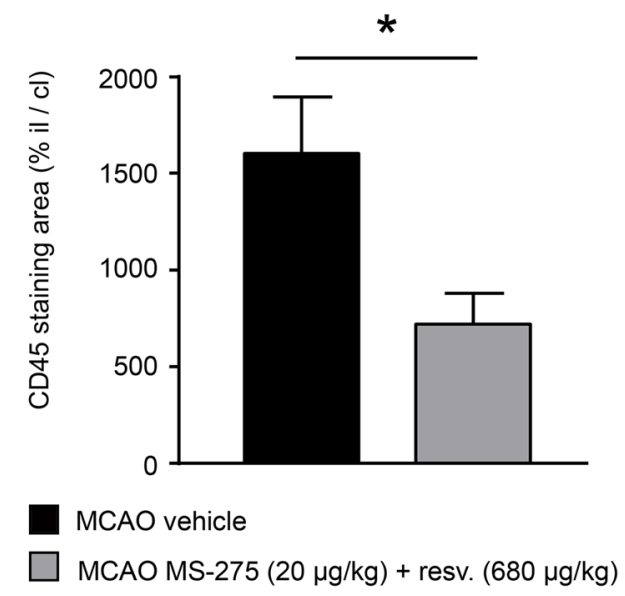

Fig. 4 The combination of MS-275 and resveratrol reduces immunoreactivity for CD45 1 day after pMCAO. (a) Representative images of CD45 immunoreactivity in the peri-infarct area of brain sections obtained from the contralateral and ipsilateral hemispheres of MCAO vehicle, and from the ipsilateral hemisphere of MCAO MS-275 (20 $\mu \mathrm{g} / \mathrm{kg})+$ resveratrol $(680 \mu \mathrm{g} / \mathrm{kg})$ mice. Scale bar: $120 \mu \mathrm{m}$. (b) Quantification of CD45 immunostaining displays an effect of MS-275 and resveratrol treatment in reducing leukocytes infiltration. Data (means \pm s.e.m. of 36-48 frames/mouse, $n=3$ ) are expressed as CD45 positive area in the ipsilateral hemisphere/ CD45 positive area in the contralateral hemisphere. Mann Whitney test, ${ }^{*} p<0.05$. 

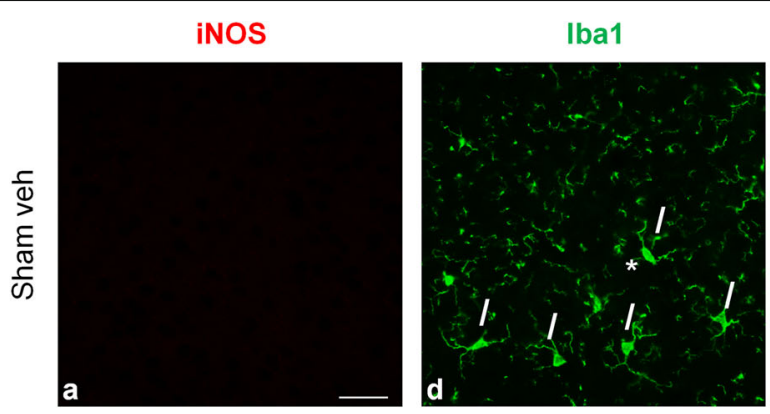

iNOS / Iba1 / Hoechst
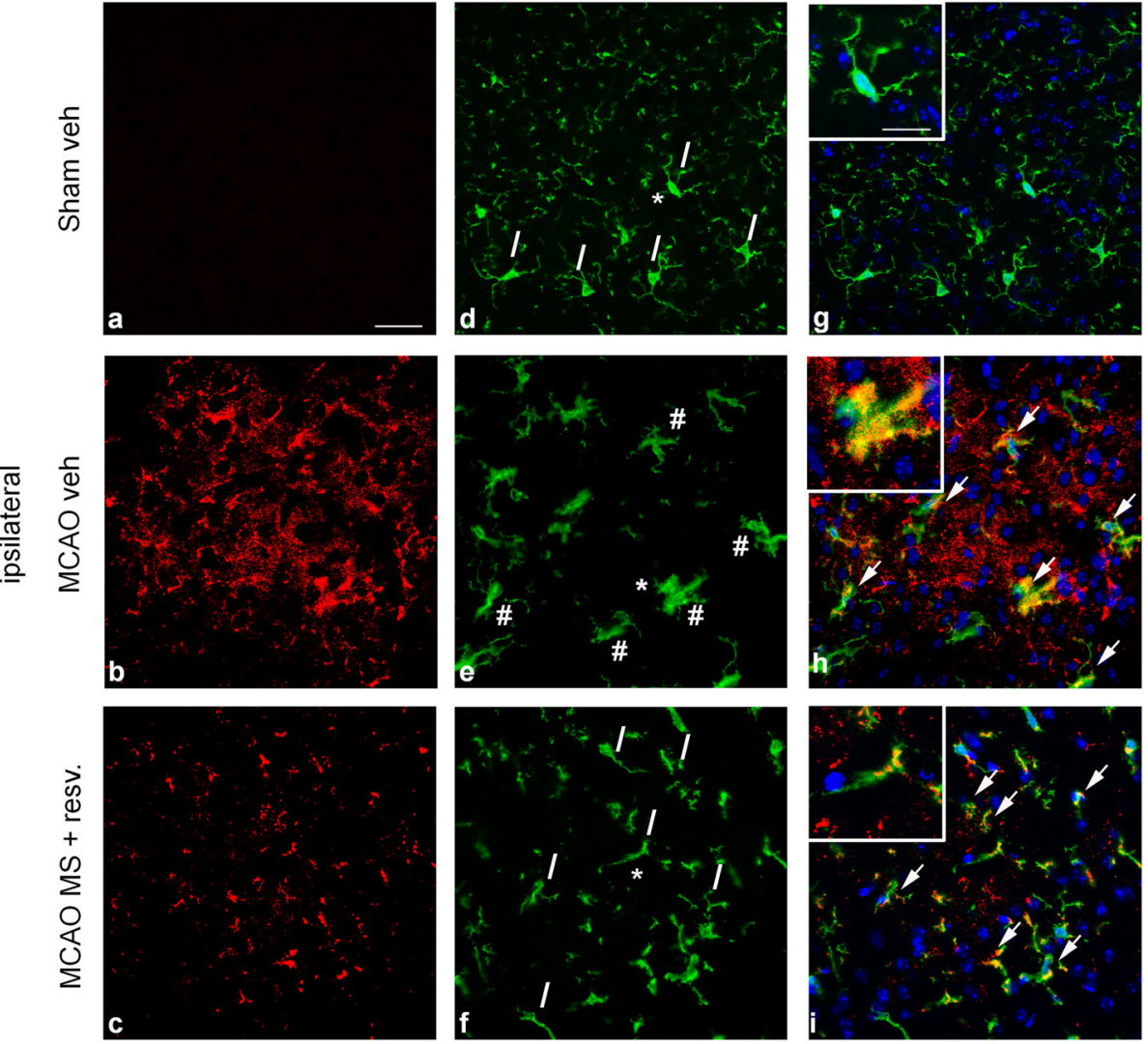

Fig. 5 The combination of MS-275 and resveratrol reduces immunoreactivity for iNOS and Iba1 1day after pMCAO. (a-i) Representative images of iNOS (red) and lba1 (green) immunofluorescence with Hoechst (blue) staining in the peri-infarct area of brain sections obtained from the ipsilateral hemispheres of $(\mathbf{a}, \mathbf{d}, \mathbf{g})$ sham vehicle, (b, e, h) MCAO vehicle, and $(\mathbf{c}, \mathbf{f}, \mathbf{i})$ MCAO MS-275 $(20 \mu \mathrm{g} / \mathrm{kg})+$ resveratrol $(680 \mu \mathrm{g} / \mathrm{kg}) \mathrm{mice}$. \# = bushy lba1+ cells, / = ramified Iba 1+ cells. Arrows represent sites of co-reactivity to iNOS and Iba1. Asterisks denote area shown in insets in higher magnification. Images are representative of 3 animals per group. Scale bars: in $\mathbf{a}=50 \mu \mathrm{m}$ for $(\mathbf{a}-\mathbf{i})$; in the inset in $\mathbf{g}=20 \mu \mathrm{m}$ for the insets in $\mathbf{g}, \mathbf{h}$ and $\mathbf{i}$.

to iNOS and CD68, as well as with the recovery of a "resting-like" ramified shape.

Treatment with MS-275 and resveratrol prevented toxicity and expression of inflammation markers in mixed glial cultures incubated with conditioned medium from neurons subjected to OGD

In order to study the direct effect of the combination of MS-275 and resveratrol on microglial activation secondary to neuronal damage, we incubated primary mixed glial cells, or pure astrocytes, overnight with conditioned medium, obtained from neurons exposed to OGD (NCMOGD), as well as from control neurons (NCM-control). The release of LDH, a marker of cellular injury, was significantly higher in mixed glial cells exposed to NCMOGD when compared to control cultures (Fig. 7a). To identify possible direct effects of the combination of MS275 and resveratrol in quenching microglial activation, we tested the treatment condition already shown to elicit synergistic neuroprotection in cortical neurons exposed to
OGD [15]. The combination of MS-275 $(0.1 \mu \mathrm{M})$ and resveratrol $(3 \mu \mathrm{M})$, added concomitantly with the exposure to NCM-OGD, significantly decreased the LDH release (Fig. 7a).

By contrast, overnight incubation of pure astrocytes with NCM-OGD did not induce significant release of LDH (Fig. 7b), suggesting that microglia present in mixed glial cultures are crucial sensors of dangerassociated molecular patterns (DAMPs) released by damaged neurons.

After incubation with NCM-OGD in mixed glial cultures, both Iba1+ microglia and GFAP+ astrocytes displayed larger bodies and thicker processes, that are morphological signs of activation (Fig. 7c). Moreover, mainly in astrocytes, but also in microglia, iNOS immunoreactivity greatly increased after exposure to NCM-OGD (Fig. 7d). The treatment with MS-275 and resveratrol reduced both the signs of glial cells activation (Fig. 7c) and the iNOS immunoreativity (Fig. 7d). 


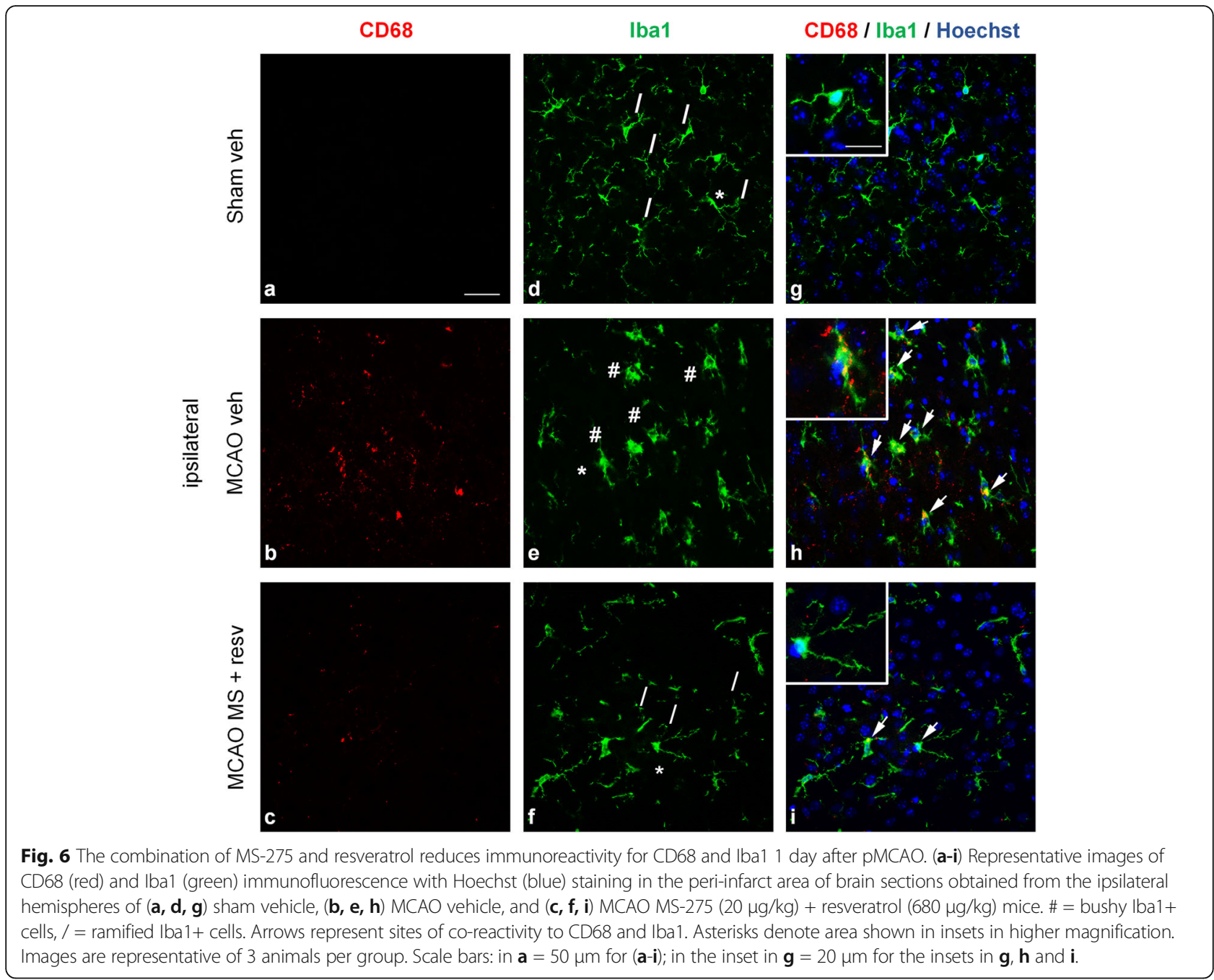

\section{Discussion}

The first aim of our work was to investigate the capability of MS-275 and resveratrol to elicit a synergistic neuroprotection when administrated in combination in a mouse model of pMCAO. Analysis of the infarct volume 2 days after pMCAO showed that a single post-insult treatment with the combination of MS-275 $(20 \mu \mathrm{g} / \mathrm{kg})$ and resveratrol $(680 \mu \mathrm{g} / \mathrm{kg})$ was neuroprotective when compared to vehicle, or administration of individual drugs administered separately. These data were accompanied by the improvement in the corner and latencyto-move trials, two established tests to assess sensorimotor function in $\mathrm{pMCAO}$-induced neurological deficits [30]. These data, supporting the valuable effect of MS275 and resveratrol in the acute treatment of ischemic stroke [15], are compatible with evidence of protective activity elicited by HDACi alone, though at a 100-fold higher dose, in models of brain ischemia [17, 18, 21].

In recent years, a body of evidence has shown that pMCAO induces a dual-phase activation of microglia/ macrophages that evolve with the time: microglia/ macrophage expressing markers for either alternative or classical activation state are induced at 24 hours and further increase at later stages [32]. We investigated the effect of the synergistic combination of MS-275 and resveratrol on the inflammatory transcription 1 day after pMCAO. At that time, both pro- (Il6, Nos 2 and Illb) and anti-inflammatory markers (Mrc1 and Ym1) appeared increased. This is in agreement with published data describing the inflammatory response observed 15 hours after the onset of pMCAO [49]. MS-275 and resveratrol-treated animals showed a lesser increase of pro-inflammatory markers, particularly a reduced transcription of Nos2, in agreement with data showing that HDACi, such as valproic acid and sodium butyrate, exhibit anti-inflammatory effects in pMCAO [50]. In our case, however, treatment with MS-275 and resveratrol also decreased the rise of anti-inflammatory markers, suggesting a general inhibitory effect on the inflammatory reaction, including the microglia/macrophage 

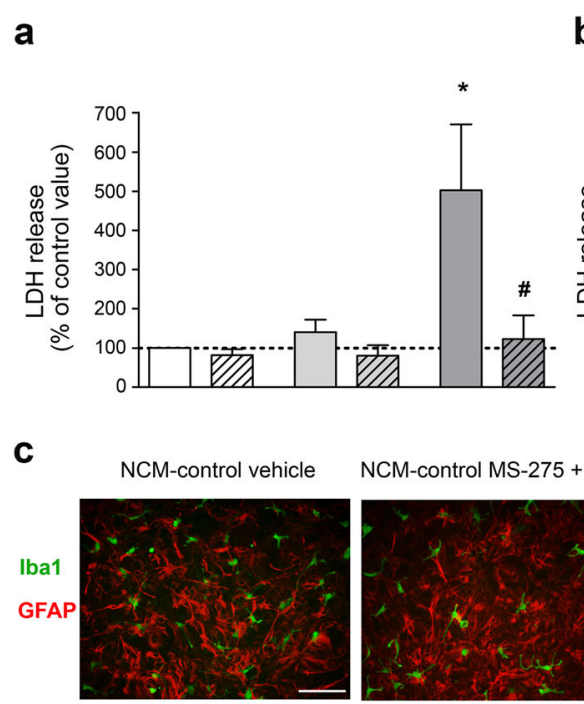

d

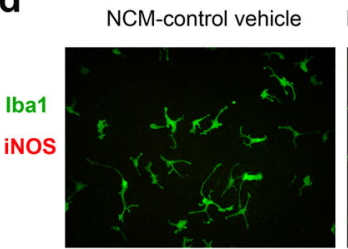

b

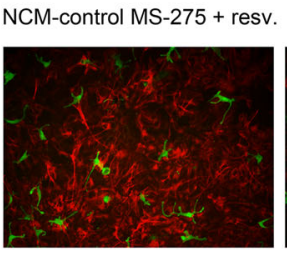

NCM-control MS-275 + resv.

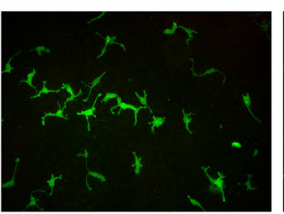

$\square$ control vehicle

control MS-275 $(0,1 \mu \mathrm{M})+$ resv. $(3 \mu \mathrm{M})$

$\square$ NCM-control vehicle

NCM-control MS-275

NCM-control MS-275
NCM-OGD vehicle

$\triangle \mathrm{NCM}-\mathrm{OGD}$ MS-275 $(0,1 \mu \mathrm{M})+$ resv. $(3 \mu \mathrm{M})$

Fig. 7 The combination of MS-275 and resveratrol reduces microglia toxicity and activation promoted by NCM. (a) LDH release from mixed glial cultures exposed to NCM. Mixed glial cultures were incubated overnight with NCM obtained from neurons subjected to OGD (NCM-OGD) or from control neurons (NCM-control). NCM-OGD induced significant toxicity in glial cells. The association of MS-275 (0.1 $\mu \mathrm{M})$ and resveratrol ( $3 \mu \mathrm{M})$ significantly reduced the cellular damage mediated by NCM-OGD. Each value is expressed as the mean \pm s.e.m. of 5 different replicate experiments, each performed in triplicate. ${ }^{*} p<0.05$, one-way ANOVA followed by Tukey multiple comparison test. (b) LDH release from pure astrocyte cultures exposed to NCM. Primary astrocytes were incubated overnight with NCM-OGD or NCM-control. The exposure to NCM did not increase the release of $L D H$ from astrocytes. Each value is expressed as the mean \pm s.e.m. of 3 different replicate experiments, each performed in triplicate. $p>0.05$, one-way ANOVA followed by Tukey multiple comparison test. (c, d) Representative images of (c) Iba1 (green) and GFAP (red) and (d) Ibal (green) and iNOS (red) immunofluorescence staining on mixed glial cells incubated with NCM-OGD or NCM-control. Cultures incubated overnight with NCM-OGD displayed increased immunofluorescence for iNOS and morphological signs of activation in GFAP+ astrocytes and Iba1+ microglial cells. In (d), arrows in NCM-OGD image represent sites of co-reactivity to iNOS and Iba1. Treatment with MS-275 $(0.1 \mu \mathrm{M})$ and resveratrol $(3 \mu \mathrm{M})$ reduced reactivity to iNOS, and promoted recovery of the signs of glial cells activation in Iba1+ and GFAP+ cells. Images are representative of 3 experiments per group. Scale bar: in $\mathbf{c}=50 \mu \mathrm{m}$ for $\mathbf{c}$ and $\mathbf{d}$.

activation [49]. The anti-inflammatory effect of MS-275 and resveratrol waned at a longer post-ischemic time. At 7 days, the treatment did not reduce the sustained increase of pro-inflammatory Nos 2 and Fcgr3, as well as the increase of anti-inflammatory $M r c 1, T g f b 1$ and Trem 2 transcripts. Yet, the treatment enhanced the expression of $F g c r 2 b$ and alternative activation markers of microglia/macrophages, Ym1 and Arg1. In view of the opposite regulation elicited by the Fgcr immunocomplex receptors on immune cells, Fcgr3 stimulatory and Fcgr $2 b$ inibitory of the pro-inflammatory state [37], it can be assumed that MS-275 and resveratrol enhanced the polarization of immune cells, including microglia/macrophages, toward an anti-inflammatory mode at a later post-ischemic phase. A condition to which peripheral immune cells as monocyte-derived macrophages highly contribute [41].
In line with the drug-induced reduction of inflammatory profile and infarct volume, the analysis of the area of CD45-positive cells in treated mice showed a reduced leukocytes infiltration 1 day after MCAO. No change was evident in the enhanced infiltration at 7 days after the ischemic insult in treated mice.

This result suggests that, although ineffective in reducing the upregulation of inflammatory gene transcripts and leukocytes brain invasion at this longer interval (7 days), the drug single administration was effective in driving later infiltrating monocytes toward a protective, anti-inflammatory profile. An evidence that makes compelling the study of repeated administrations of the drugs after pMCAO.

To assess whether the results observed at 24 hours were due to an epigenetic effect, we performed a ChIP analysis at the Nos 2 promoter, a NF- $\mathrm{kB}$ target gene [43] 
whose transcription was inhibited by the treatment. The assay revealed a strong decrease in RelA binding and $\mathrm{H} 3$ acetylation at the Nos 2 promoter in ipsilateral hemispheres from mice treated with MS-275 and resveratrol after the insult, compared to vehicle-treated animals. Another NF- $\mathrm{kB}$ target gene that was increased after pMCAO was Il6, but the ChIP results showed that RelA binding to its promoter did not increase after the insult, leading us to conclude that, at the tested time point, NF$\kappa \mathrm{B}$ is dispensable for Il6 expression. This in line with evidence that the transcription of $I l 6$ in brain ischemia is also regulated by CREB [51], C/EBP $\beta$ [52], and AP-1 [53], all transcription factors shown to cooperate with NF-kB in controlling the Il6 transcript levels [54].

These results led us to hypothesize that microglia could be less activated. Although our experimental design did not allow us to distinguish between microglia and peripheral macrophages, in the peri-infarct area most of the Iba1+ cells are resident microglia 1 day after permanent ischemia [55]. With the aim of assessing the effect of the drug combination on the activation state of microglia/macrophages, we performed a double immunofluorescence labeling of Iba1 with iNOS and CD68, respectively a pro-inflammatory and a phagocytosis markers. Our examination revealed Iba1+ cells on the peri-infarct area with mostly bushy/hypertrophic and ramified morphology, whereas round Iba1+ cells were rarely seen. The presence of thinly ramified Iba1+ cells, morphologically similar to those present in sham mice, was evident in the peri-infarct area of drug-treated animals, compared to vehicle-treated. This suggests that the administration of MS-275 and resveratrol decreases the activation of microglia/macrophages [56].

The combination of MS-275 and resveratrol reduced the immunoreactivity to iNOS, though this reduction is mainly seen in Iba1- cells. Other cell types, such as astrocytes, express this marker [44]. Iba1+/iNOS+ cells presented ramified and bushy morphologies, in agreement with recent published data [42].

Acute treatment of ischemic mice resulted in lowered immunoreactivity for CD68+ phagocytic cells. Phagocytosis in cerebral ischemia is a complex phenomenon and is not clearly linked to a specific pro-/anti-inflammatory state [57], or beneficial/deleterious effects [58]. Alike iNOS, CD68 was detected in Iba1- cells, which could be infiltrating phagocytic CD68+/Iba1- neutrophils [47], while CD68+/Iba1+ were ramified and bushy cells, as was previously shown [42]. We found that, at 1 day post-pMCAO, the immunoreactivity for MRC1 did not change between sham, vehicle- or drug-treated animals. This seems in agreement with recent published data, at 1 day after pMCAO, showing that co-positivity for MRC1 and Iba1 is detectable exclusively in round, ameboid cells [42]. It should be noted that, although significant, the increase of the mRNA of MRC1 at 1 day is minimal. Therefore, this immunofluorescence finding is in line with the low-grade expression of MRC1 detected after pMCAO.

Our results show that treatment with MS-275 and resveratrol decreases the pro-inflammatory activation of Iba1+ microglia/macrophages in the peri-infarct area, by reducing their immunoreactivity to iNOS and CD68.

The last step was to confirm whether the antiinflammatory effect seen in vivo was neuron-mediated or if the treatment could elicit a direct antiinflammatory effect on glial cells. With the intent of using a cellular model that could mirror some of the complexity of the post-ischemic environment and players, primary cultures of mixed glial cells and pure astrocytes were incubated overnight with MS-275 and resveratrol in a NCM from neurons exposed to OGD. The results showed that microglia are the first cells to sense and respond to damaged neurons, because only when they were present, in mixed glial cell culture, the exposure to NCM-OGD increased the release of LDH. The decrease in LDH release from mixed glial cells incubated with MS-275 and resveratrol demonstrates that the epigenetic drugs can directly target glial cells.

The increase in immunoreactivity for iNOS, seen after the incubation of mixed glial cell with NCM-OGD, is in line with RelA binding and histone $\mathrm{H} 3$ acetylation at the Nos 2 promoter observed in the pMCAO model. Noteworthy, iNOS activation was not observed exclusively in Iba1+ cells, but mostly in Iba1- cells, the astrocytes. We can assume that, although microglia are the first sensors of neuronal distress mediated by DAMPs, they rapidly communicate with other cells, disseminating the "message".

\section{Conclusion}

By combining these with previous findings [15], we can conclude that the aberrant acetylation of RelA, responsible for the pro-apoptotic transcription following noxious brain ischemia, is reverted by a single post-insult administration of MS-275 and resveratrol. The change in RelA acetylation hampers RelA interaction with the Nos 2 promoter and, by shifting the binding of transcription factor from the Bim to $B c l-\chi_{L}$ gene, concomitantly reduces the pro-inflammatory transcription and promotes the anti-apoptotic one. MS-275 and resveratrol synergistically reduced brain damage and neurological deficits, showing to be more efficient than individual drugs used at 100-fold higher doses [15]. We can assume that the neuroprotective activity observed 2 days after pMCAO occurred through different, complementary mechanisms. The drugs induced neuron survival and, both directly and indirectly, reduced pro-inflammatory activation of microglia/macrophages. Along with the 
inhibition of the early inflammatory response, the treatment also limited the leukocytes infiltration thus quenching further the expression of pro-inflammatory genes. The beneficial effects of a single administration was not permanent, although it could drive the later infiltrating monocytes toward a protective antiinflammatory phenotype.

Our data supports the use of MS-275 and resveratrol in treating acute ischemic stroke at a very low doses, with less risk of side effects. Future experiments testing repeated administration of these drugs during the subacute phase will unveil the possibility to maximize the functional recovery after stroke.

\section{Supplementary Information}

The online version contains supplementary material available at https://doi. org/10.1186/s12974-020-02028-4.

Additional file 1. Inflammatory and microglia/macrophage mRNA expression profile in the ipsilateral hemisphere 7 days after pMCAO. (a-I) Quantification of 116, Nos2, II1b, Fcgr3, Fcgr2b, Mrc1, Arg1, Ym1, Tgfb1 and Trem 2 by qRT-PCR in the ipsilateral hemisphere 7 days after pMCAO. Seven days since stroke induction, expression of (g) $\operatorname{Arg} 1$ and (h) Ym 1 was not different from sham vehicle group. Transcript levels of (b) Nos2, (d) Fcgr3, (f) Mrc1, (i) Tgfb1 and (I) Trem2 were significantly higher when compared to sham vehicle group, while (c) $111 b$ and (e) Fcgr2b showed an upward trend. Expression of (a) $1 / 6$ was significantly lower. Treatment with MS-275 and resveratrol did not increase the expression of (a) 116 , nor reduce the transcript levels of (b) Nos2, (d) Fcgr3, (f) Mrcl, (i) Tgfb1 and (I) Trem2. Instead, the treatment enhanced the expression of (e) Fcgr2b, (g) Arg1 and (h) Ym1. One-way ANOVA followed by Holm-Šídák test, ${ }^{\circ}$ trend, ${ }^{*} p<0.05,{ }^{* *} p<0.01,{ }^{* * *} p<0.001,{ }^{* * * *} p<0.0001$ vs. sham vehicle; \#trend, $\S \S \S p<0.001$ vs. MCAO vehicle. Data (means \pm s.e.m., $n=6$ ) are expressed as fold changes over values obtained in ipsilateral hemispheres from sham operated mice.

Additional file 2. CD45 immunoreactivity in the peri infarct area 7 days after pMCAO. (a) Representative images of CD45 immunoreactivity in the peri-infarct area of brain sections obtained from the controlateral and ipsilateral hemispheres of MCAO vehicle, and from the ipsilateral hemisphere of MCAO MS-275 $(20 \mu \mathrm{g} / \mathrm{kg})+$ resveratrol $(680 \mu \mathrm{g} / \mathrm{kg})$ mice. (b) Quantification of CD45 immunostaining shows the absence of MS-275 and resveratrol treatment effect in reducing leukocytes infiltration. Data (means \pm s.e.m. of 36-42 frames/mouse, $n=3$ ) are expressed as CD45 positive area in the ipsilateral hemisphere/ CD45 positive area in the contralateral hemisphere. Scale bar: $120 \mu \mathrm{m}$. Mann Whitney test, $\mathrm{p}>0.05$.

Additional file 3. MRC1 and Ibal expression in the peri infarct area 1 day after MCAO (a-i) Representative images of MRC1 (red) and Iba1 (green) immunofluorescence with Hoechst (blue) staining on brain sections obtained from the ipsilateral hemispheres of $(\mathbf{a}, \mathbf{d}, \mathbf{g})$ sham vehicle, (b, e, h) MCAO vehicle, and (c, f, i) MCAO MS-275+resv. mice. \# = bushy $\mathrm{Iba} 1+$ cells, $/=$ ramified Ibal+ cells. Arrows represent sites of Coreactivity to MRC1 and Iba1. Asterisks denote area shown in insets in higher magnification. Images are representative of 3 animals per group. Scale bars: in $\mathbf{a}=50 \mu \mathrm{m}$ for $(\mathbf{a}-\mathbf{i})$; in the inset in $\mathbf{g}=20 \mu \mathrm{m}$ for the insets in $\mathbf{g}, \mathbf{h}$ and $\mathbf{i}$

\section{Abbreviations}

AMPK: AMP-activated kinase; ChIP: chromatin immunoprecipitation; DAMPs: danger associated molecular patterns; DMSO: dimethylsulfoxide; HATs: histone acetyltransferases; HDACi: histone deacetylase inhibitors; LDH: lactate dehydrogenase; NCM: neuron-derived conditioned medium; NFkB: nuclear factor-kappa B; OGD: oxygen glucose deprivation; PMCAO: permanent middle cerebral artery occlusion; qRT-PCR: quantitative reverse transcription-polymerase chain reaction; rtPA: recombinant tissue plasminogen activator; tMCAO: transient middle cerebral artery occlusion

\section{Acknowledgements}

Not applicable.

\section{Authors' contributions}

MM and VP: designed and performed experiments, contributed to study design, collected and analyzed data, prepared figures and wrote manuscript; EP: analyzed the data and wrote manuscript; AB: contributed to study design and supervised studies; MB: performed experiments; SR: contributed to study design and performed experiments; MS: contributed to study design, supervised studies and provided financial support; MP: designed and supervised studies, prepared final manuscript, provided financial support for all studies. All authors read and approved the final manuscript.

\section{Funding}

This research was supported by Seventh Framework Programme (FP7 Project No. 607962)

\section{Availability of data and materials}

The datasets and materials used and/or analysed during the current study are available from the corresponding author on reasonable request.

\section{Ethics approval and consent to participate}

The animal studies were performed according with the Directive 2010/63/EU on the protection of animals used for scientific purposes and were approved by the German and Italian animal welfare authorities (Ministerium für Energiewende, Landwirtschaft, Umwelt, Natur und Digitalisierung, Kiel, Germany; Ministero della Salute, Roma, Italy).

\section{Consent for publication}

Not applicable.

\section{Competing interests}

The authors declare that they have no competing interests.

\section{Author details}

'Division of Pharmacology, Department of Molecular and Translational Medicine, University of Brescia, Viale Europa 11, 25123 Brescia, Italy. ${ }^{2}$ Institute for Experimental and Clinical Pharmacology and Toxicology, University of Lübeck, Lübeck, Germany.

Received: 5 August 2020 Accepted: 9 November 2020

Published online: 27 November 2020

\section{References}

1. Hankey G. Stroke Lancet. 2017;389:641-54.

2. Feigin VL, Norrving B, Mensah GA. Global burden of stroke. Circ Res. 2017; 120:439-48.

3. Donnan GA, Fisher M, Macleod M, Davis SM. Stroke. Lancet. 2008;371: 1612-23.

4. Fisher M, Saver JL. Future directions of acute ischaemic stroke therapy. Lancet Neurol. 2015:14:758-67.

5. Dirnagl U, ladecola C, Moskowitz MA. Pathobiology of ischaemic stroke: an integrated view. Trends Neurosci. 1999:22:391-7.

6. Mattson MP, Meffert MK. Roles for NF-kappaB in nerve cell survival, plasticity, and disease. Cell Death Differ. 2006;13:852-60.

7. Kaltschmidt B, Kaltschmidt C. NF-KappaB in long-term memory and structural plasticity in the adult mammalian brain. Front Mol Neurosci. 2015; 8:69.

8. Mattson MP, Camandola S. NF-kappaB in neuronal plasticity and neurodegenerative disorders. J Clin Invest. 2001;107:247-54

9. Lanzillotta A, Porrini V, Bellucci A, Benarese M, Branca C, Parrella E, et al. NF$\mathrm{KB}$ in innate neuroprotection and age-related neurodegenerative diseases. Front Neurol. 2015;6:98.

10. Inta I, Paxian S, Maegele I, Zhang W, Pizzi M, Spano P, et al. Bim and Noxa are candidates to mediate the deleterious effect of the NF-kappa B subunit RelA in cerebral ischemia. J Neurosci. 2006;26:12896-903.

11. Schneider A, Martin-Villalba A, Weih F, Vogel J, Wirth T, Schwaninger M. NFkappaB is activated and promotes cell death in focal cerebral ischemia. Nat Med. 1999;5:554-9. 
12. Pizzi M, Boroni F, Bianchetti A, Moraitis C, Sarnico I, Benarese M, et al. Expression of functional NR1/NR2B-type NMDA receptors in neuronally differentiated SK-N-SH human cell line. Eur J Neurosci. 2002;16:2342-50.

13. Pizzi M, Sarnico I, Boroni F, Benarese M, Steimberg N, Mazzoleni G, et al. NFkappaB factor c-Rel mediates neuroprotection elicited by mGlu5 receptor agonists against amyloid beta-peptide toxicity. Cell Death Differ. 2005;12: 761-72

14. Ingrassia R, Lanzillotta A, Sarnico I, Benarese M, Blasi F, Borgese L, et al. 1B/ (-)IRE DMT1 expression during brain ischemia contributes to cell death mediated by NF-KB/RelA acetylation at Lys310. PLoS One. 2012;7:e38019.

15. Lanzillotta A, Pignataro G, Branca C, Cuomo O, Sarnico I, Benarese M, et al. Targeted acetylation of NF-kappaB/RelA and histones by epigenetic drugs reduces post-ischemic brain injury in mice with an extended therapeutic window. Neurobiol Dis. 2013:49:177-89.

16. Lanzillotta A, Sarnico I, Ingrassia R, Boroni F, Branca C, Benarese M, et al. The acetylation of RelA in Lys310 dictates the NF-kappaB-dependent response in post-ischemic injury. Cell Death Dis. 2010;1:e96.

17. Ren $M$, Leng $Y$, Jeong $M$, Leeds PR, Chuang DM. Valproic acid reduces brain damage induced by transient focal cerebral ischemia in rats: potential roles of histone deacetylase inhibition and heat shock protein induction. J Neurochem. 2004:89:1358-67.

18. Park MJ, Sohrabji F. The histone deacetylase inhibitor, sodium butyrate, exhibits neuroprotective effects for ischemic stroke in middle-aged female rats. J Neuroinflammation. 2016;13:300.

19. Patnala R, Arumugam TV, Gupta N, Dheen ST. HDAC inhibitor sodium butyrate-mediated epigenetic regulation enhances neuroprotective function of microglia during ischemic stroke. Mol Neurobiol. 2016;54(8):6391-411.

20. Ma XH, Gao Q, Jia Z, Zhang ZW. Neuroprotective capabilities of TSA against cerebral ischemia/reperfusion injury via PI3K/Akt signaling pathway in rats. Int J Neurosci. 2015;125:140-6.

21. Wang Z, Leng Y, Tsai LK, Leeds P, Chuang DM. Valproic acid attenuates blood-brain barrier disruption in a rat model of transient focal cerebral ischemia: the roles of HDAC and MMP-9 inhibition. J Cereb Blood Flow Metab. 2011;31:52-7.

22. Wan D, Zhou Y, Wang K, Hou Y, Hou R, Ye X. Resveratrol provides neuroprotection by inhibiting phosphodiesterases and regulating the CAMP/ AMPK/SIRT1 pathway after stroke in rats. Brain Res Bull. 2016;121:255-62.

23. Agrawal M, Kumar V, Kashyap MP, Khanna VK, Randhawa GS, Pant AB. Ischemic insult induced apoptotic changes in PC12 cells: protection by trans resveratrol. Eur J Pharmacol. 2011;666:5-11.

24. Li H, Yan Z, Zhu J, Yang J, He J. Neuroprotective effects of resveratrol on ischemic injury mediated by improving brain energy metabolism and alleviating oxidative stress in rats. Neuropharmacology. 2011;60:252-8.

25. Yu P, Wang L, Tang F, Zeng $L$, Zhou L, Song $X$, et al. Resveratrol pretreatment decreases ischemic injury and improves neurological function via sonic hedgehog signaling after stroke in rats. Mol Neurobiol. 2017:54: 212-26.

26. Schiaffino L, Bonafede R, Scambi I, Parrella E, Pizzi M, Mariotti R. Acetylation state of RelA modulated by epigenetic drugs prolongs survival and induces a neuroprotective effect on ALS murine model. Sci Re. 2018;8(1):12875.

27. Zhou W, Liesz A, Bauer H, Sommer C, Lahrmann B, Valous $N$, et al. Postischemic brain infiltration of leukocyte subpopulations differs among murine permanent and transient focal cerebral ischemia models. Brain Pathol. 2013;23(1):34-44

28. Fluri F, Schuhmann MK, Kleinschnitz C. Animal models of ischemic stroke and their application in clinical research. Drug Des Devel Ther. 2015;9:3445-54

29. McBride DW, Zhang JH. Precision stroke animal models: the permanent MCAO model should be the primary model. Not Transient MCAO Trans Stroke Res. 2017. https://doi.org/10.1007/s12975-017-0554-2.

30. Rahman M, Muhammad S, Khan MA, Chen H, Ridder DA, Müller-Fielitz H, et al. The $\beta$-hydroxybutyrate receptor HCA2 activates a neuroprotective subset of macrophages. Nat Commun. 2014;5:3944.

31. Herrmann O, Baumann B, de Lorenzi R, Muhammad S, Zhang W, Kleesiek J, et al. IKK mediates ischemia-induced neuronal death. Nat Med. 2005;11(12): 1322-9.

32. Perego C, Fumagalli S, De Simoni MG. Temporal pattern of expression and colocalization of microglia/macrophage phenotype markers following brain ischemic injury in mice. J Neuroinflammation. 2011;8:174.

33. Faggi L, Porrini V, Lanzillotta A, Benarese M, Mota M, Tsoukalas D, Parrella E, Pizzi M. A Polyphenol-Enriched Supplement Exerts Potent Epigenetic-
Protective Activity in a Cell-Based Model of Brain Ischemia. Nutrients. 2019; 11(2), pii: E345.

34. Porrini V, Lanzillotta A, Branca C, Benarese M, Parrella E, Lorenzini L, et al. CHF5074 (CSP-1103) induces microglia alternative activation in plaque-free Tg2576 mice and primary glial cultures exposed to beta-amyloid Neuroscience. 2015:302:112-20.

35. Schildge S, Bohrer C, Beck K, Schachtrup C. Isolation and Culture of Mouse Cortical Astrocytes. J. Vis. Exp. 2013; 71. pii: 50079.

36. Lambertsen KL, Biber K, Finsen B. Inflammatory cytokines in experimental and human stroke. J Cereb Blood Flow Metab. 2012:32:1677-98.

37. Chauhan P, Hu S, Sheng WS, Prasad S, Lokensgard JR. Modulation of microglial cell Fcy receptor expression following viral brain infection. Sci Rep. 2017:7:41889.

38. ladecola C, Anrather J. The immunology of stroke: from mechanisms to translation. Nat Med. 2011:17:796-808.

39. Hu X, Leak RK, Shi Y, Suenaga J, Gao Y, Zheng P, et al. Microglial and macrophage polarization—-new prospects for brain repair. Nat Rev Neurol. 2015;11:56-64

40. Kawabori M, Kacimi R, Kauppinen T, Calosing C, Kim JY, Hsieh CL, et al. Triggering receptor expressed on myeloid cells 2 (TREM2) deficiency attenuates phagocytic activities of microglia and exacerbates ischemic damage in experimental stroke. J Neurosci. 2015;35:3384-96.

41. Wattananit S, Tornero D, Graubardt N, Memanishvili T, Monni E, Tatarishvili J, et al. Monocyte-derived macrophages contribute to spontaneous long-term functional recovery after stroke in mice. J Neurosci. 2016;36(15):4182-95.

42. Perego C, Fumagalli S, Zanier ER, Carlino E, Panini N, Erba E, et al. Macrophages are essential for maintaining a M2 protective response early after ischemic brain injury. Neurobiol Dis. 2016;96:284-93.

43. Li Q, Verma IM. NF-kappaB regulation in the immune system. Nat Rev Immunol. 2002:2:725-34.

44. Saha RN, Pahan K. Regulation of inducible nitric oxide synthase gene in glial cells. Antioxid Redox Signal. 2006;8:929-47.

45. Damoiseaux JG, Döpp EA, Calame W, Chao D, MacPherson GG, Dijkstra CD. Rat macrophage lysosomal membrane antigen recognized by monoclonal antibody ED1. Immunology. 1994;83:140-7.

46. Fumagalli S, Perego C, Ortolano F, De Simoni MG. CX3CR1 deficiency induces an early protective inflammatory environment in ischemic mice. Glia. 2013:61:827-42

47. Lively S, Hutchings S, Schlichter LC. Molecular and cellular responses to Interleukin-4 treatment in a rat model of transient ischemia. J Neuropathol Exp Neurol. 2016;75:1058-71.

48. Perez-de-Puig I, Miró-Mur F, Ferrer-Ferrer M, Gelpi E, Pedragosa J, Justicia C, et al. Neutrophil recruitment to the brain in mouse and human ischemic stroke. Acta Neuropathol. 2015;129:239-57.

49. Zarruk JG, Fernández-López D, García-Yébenes I, García-Gutiérrez MS, Vivancos J, Nombela F, et al. Cannabinoid type 2 receptor activation downregulates stroke-induced classic and alternative brain macrophage/ microglial activation concomitant to neuroprotection. Stroke. 2012;43:211-9.

50. Kim HJ, Rowe M, Ren M, Hong JS, Chen PS, Chuang DM. Histone deacetylase inhibitors exhibit anti-inflammatory and neuroprotective effects in a rat permanent ischemic model of stroke: multiple mechanisms of action. J Pharmacol Exp Ther. 2007;321:892-901.

51. Nakamura K, Kamouchi M, Arimura K, Nishimura A, Kuroda J, Ishitsuka K, et al. Extracellular acidification activates CAMP responsive element binding protein via $\mathrm{Na}+/ \mathrm{H}+$ exchanger isoform 1-mediated $\mathrm{Ca}^{2+}$ oscillation in central nervous system pericytes. Arterioscler Thromb Vasc Biol. 2012;32: 2670-7.

52. Kapadia R, Tureyen K, Bowen KK, Kalluri H, Johnson PF, Vemuganti R. Decreased brain damage and curtailed inflammation in transcription factor CCAAT/enhancer binding protein beta knockout mice following transient focal cerebral ischemia. J Neurochem. 2006;98:1718-31.

53. Ishibashi N, Prokopenko O, Reuhl KR, Mirochnitchenko O. Inflammatory response and glutathione peroxidase in a model of stroke. J Immunol. 2002; 168:1926-33.

54. Poplutz MK, Wessels I, Rink L, Uciechowski P. Regulation of the Interleukin-6 gene expression during monocytic differentiation of HL-60 cells by chromatin remodeling and methylation. Immunobiology. 2014;219:619-26.

55. Tanaka R, Komine-Kobayashi M, Mochizuki H, Yamada M, Furuya T, Migita M, et al. Migration of enhanced green fluorescent protein expressing bone marrow-derived microglia/macrophage into the mouse brain following permanent focal ischemia. Neuroscience. 2003;117:531-9. 
56. Ladeby R, Wirenfeldt M, Garcia-Ovejero D, Fenger C, Dissing-Olesen L, Dalmau I, et al. Microglial cell population dynamics in the injured adult central nervous system. Brain Res Brain Res Rev. 2005;48:196-206.

57. Fumagalli S, Perego C, Pischiutta F, Zanier ER, De Simoni MG. The ischemic environment drives microglia and macrophage function. Front Neurol. 2015;6:81.

58. Brown GC, Neher JJ. Inflammatory neurodegeneration and mechanisms of microglial killing of neurons. Mol Neurobiol. 2010;41:242-7.

\section{Publisher's Note}

Springer Nature remains neutral with regard to jurisdictional claims in published maps and institutional affiliations.

Ready to submit your research? Choose BMC and benefit from:

- fast, convenient online submission

- thorough peer review by experienced researchers in your field

- rapid publication on acceptance

- support for research data, including large and complex data types

- gold Open Access which fosters wider collaboration and increased citations

- maximum visibility for your research: over $100 \mathrm{M}$ website views per year

At $B M C$, research is always in progress.

Learn more biomedcentral.com/submissions 